\title{
THE LOCATION OF STELLAR CLUSTERS IN THE BIG DENT: AN AGE GRADIENT ALONG THE Z-AXIS
}

\author{
Jesús Cabrera-Caño, ${ }^{1,2}$ Edmundo Moreno, ${ }^{3}$ José Franco, $^{3}$ and Emilio J. Alfaro ${ }^{1,4}$ \\ Received 1994 February 11; accepted 1995 January 30
}

\begin{abstract}
The ages and locations of stellar clusters and individual Cepheid stars in the depression called the "Big Dent" are analyzed. The $Z$-locations of the cluster sample display a well-defined $Z$-age stratification. The Cepheid sample has ages between 3 and $8 \times 10^{7} \mathrm{yr}$ and shows a departure from the midplane similar to the one observed for clusters of the same age range. The existence of such an age gradient seems to corroborate previous hypotheses suggesting that the star formation activity was probably triggered by the same strong perturbation which originated the depression. A model in which the Big Dent was created by a collision of a high-velocity cloud with the Galactic disk is able to reproduce the observed gradient.
\end{abstract}

Subject headings: Cepheids - Galaxy: structure - open clusters and associations: general — stars: formation

\section{INTRODUCTION}

The structure of the Galactic disk in the vicinity of the Sun, delineated by the location of stellar clusters and interstellar clouds, displays a series of large-scale features which depart more than $100 \mathrm{pc}$ from the formal midplane (see Alfaro, Cabrera-Caño, \& Delgado 1991). In particular, there is a nearby huge depression, some $1.5 \mathrm{kpc}$ in diameter and reaching some $150 \mathrm{pc}$ below the plane, called the "Big Dent." It contains relatively young stellar groups indicating that some strong perturbations probably triggered the formation of stars more than $10^{7} \mathrm{yr}$ ago. In fact, these large- $Z$ departures are sometimes associated with star-forming regions (i.e., the Gould's Belt, the Perseus complex, etc.), and some others seem to confirm largescale corrugations of the disk (e.g., Spicker \& Feitzinger 1986; Alfaro, Cabrera-Caño, \& Delgado 1992).

All these distortions require a powerful source of mechanical energy, able to displace a large section of the Galactic disk along the $Z$-axis, and some mechanisms have been proposed to explain both the observed $Z$ displacements and the different stages of star formation associated with them (see reviews by Elmegreen 1992 and Franco 1992). One obvious candidate to provide the required amount of momentum injection is the interaction between high-velocity clouds (HVCs) and the disk (e.g., Tenorio-Tagle 1980; Tenorio-Tagle et al. 1986, 1987), and this mechanism seems to be able to reproduce the location and properties of several star-forming regions: the Orion and Monoceros molecular complexes (Franco et al. 1988; Lepine \& Duvert 1994), the Big Dent (Alfaro et al. 1991), the Gould's Belt (Comerón \& Torra 1992; Lepine \& Duvert 1994), and the Perseus complex (Phelps 1992).

Here we deal with the history of star formation in the Big Dent, the largest and deepest depression in the vicinity of

\footnotetext{
${ }^{1}$ Universidad de Sevilla, Apdo. Correos 1065, Sevilla, 41080 Spain; jesus@obelix.cica.es. Also Instituto de Astrofisica de Andalucía (CSIC), Granada, Spain.

${ }^{2}$ Instituto de Astronomía, UNAM, Apdo. Postal 70-264, México D.F., México; edmundo@astroscu.unam.mx.

${ }^{3}$ Instituto de Astronomía, UNAM, Apdo. Postal 70-264, México D.F., México; pepe@astroscu.unam.mx.

${ }^{4}$ Instituto de Astrofísica de Andalucía (CSIC), Apdo. Correos 3004, Granada, 18080 Spain; emilio@iaa.es. Also Boston University.
}

the Sun, and we analyze the existence and possible origin of a $Z$-age gradient in this supercomplex. The large range in $Z$-locations for the stellar clusters belonging to the Big Dent (about $200 \mathrm{pc}$ ) allows us to obtain a clear separation between stellar groups with different ages. The resulting $Z$-age relationship is then compared with the one predicted by a simple semianalytical treatment, including the effects of interstellar drag and radiation pressure, of the evolution of a cloud formed in a HVC-disk collision. It is assumed that the shocked layer becomes a star-forming cloud some time after passage through the plane, and the model provides the locations, as a function of time, of this star-forming cloud and the newly formed stars. The newly formed stars are not affected by the radiation pressure and the interstellar drag and can detach from their parent cloud generating, in a series of different episodes of star formation, the $Z$-age correlation.

The paper has been structured in four sections. The data samples used to analyze the temporal history of the Big Dent are described in $\S 2$. The selection of the open cluster subsample used to perform this analysis and the resulting $Z$-age gradient are described in $\$ 2.1$ and 2.2 , respectively. The vertical structure of Cepheids in the region is presented in $\S 2.3$, and the possible effects of extinction in the $Z$-age relation in the Big Dent are discussed in $\S 2.4$. The physics involved in the HVCdisk collision and the mean guidelines of the model are described in $\S 3$. Finally, $\S 4$ contains a brief discussion.

\section{THE $Z$-AGE GRADIENT IN THE BIG DENT}

Suggestions for the existence of a $Z$-age gradient in several star-forming regions have been claimed in recent years. Sitnik (1989), from a study of the spatial distribution of open clusters in the Perseus complex, pointed out the possible existence of a $Z$-age correlation in this Galactic region. Later, Phelps (1992) in a careful analysis of the location and age of young star clusters, along with the distribution of molecular and atomic gas in the same complex, reached a similar conclusion and proposed an HVC-disk collision as the most plausible origin for this structure. A topography of the Galactic plane, as depicted by a sample of Cepheid stars, was obtained by Berdnikov \& Efremov (1993) and corroborated the existence of a depression located at the position of the Big Dent but derived a smaller departure from the Galactic plane $(Z=-50 \mathrm{pc})$ than 
the one shown by the young open clusters. They pointed out that this difference could be explained by the difference in age of the samples, implicitly suggesting the existence of a relationship between $Z$ and age for the Big Dent.

\subsection{Data}

Our aim here is to obtain a reliable distribution of $Z$-coordinates and ages for objects located within the Big Dent. Young open clusters and Cepheid stars seem to be the best type of objects to trace a possible relationship between age and location. These objects have been used in several studies of Galactic structure (e.g., Lyngå 1982, 1985; Janes, Tilley, \& Lyngå 1988) and in the first studies of the Galactic distribution of star complexes (Efremov 1978a; Efremov \& Sitnik 1988). They have also been a very useful tracer to delineate the vertical structure of the disk in the solar vicinity (Alfaro et al. 1991, 1992; Berdnikov \& Efremov 1993).

An almost complete catalog of known open clusters (containing about 1200 objects) has been compiled by Lyngå $(1981,1987)$, and, more recently, a more homogeneous data set was published by Loktin \& Matkin (1990) and Loktin, Matkin, \& Gerasimenko (1994; hereafter LMG94). Both samples are available in machine-readable form, and they satisfy two important conditions: (1) They provide distances and ages, and (2) they contain a sufficient number of objects to perform an adequate analysis. The main restriction of the Lyngå catalog, however, is the lack of homogeneity in the compiled data (the distances and ages were determined by a series of different authors, using different techniques and instrumentations). The more homogeneous catalog (LMG94), although not as extensive as the Lyngå compilation, is more reliable for Galactic studies, and we use it here to perform the analysis of the agelocation relationship in the Big Dent.

Cepheid stars, on the other hand, represent a fundamental component of stellar complexes, and any correlation between ages and locations should also be reflected in the distribution of Cepheids. Two homogeneous catalogs of known Cepheid stars have been published by Berdnikov (1987, hereafter B87) and Caldwell \& Coulson (1987, hereafter CC87). These authors compiled periods and broadband photometry for a large sample of Galactic Cepheids, and both catalogs have already been used in galactic structure studies. CC87 derived the solar galactocentric radius and Oort's $A$ constant from their own compilation, and Berdnikov \& Efremov (1993) analyzed the vertical structure of the Galactic disk in the vicinity of the Sun from the B87 data. Finally, to have the required data, the Cepheid ages were obtained from the period-age relationship given by Efremov (1978b).

\subsection{The Z-Age Gradient}

All clusters with ages smaller than $3 \times 10^{8} \mathrm{yr}$ and located in the direction of the Big Dent were extracted from the LMG94 catalog. The variables $D$ (distance from the Sun) and $l$ and $b$ (Galactic longitude and latitude) were then transformed into a Cartesian coordinate system $(X, Y, Z)$ centered on the Sun. The $X$-axis takes positive values in the direction of Galactic rotation, the $Y$-axis is directed toward the Galactic anticenter, and the $Z$-axis is positive in the north Galactic hemisphere. A spatial box containing the Big Dent is then defined with $-2.5<X<0.3 \mathrm{kpc}$ and $0.2<Y<2.5 \mathrm{kpc}$ (see Alfaro et al. 1991). Figure 1 shows a plot of $Z$ versus log age, and the resulting least-squares linear fit, for the 34 clusters contained within that box. A clear trend is apparent in the figure, and

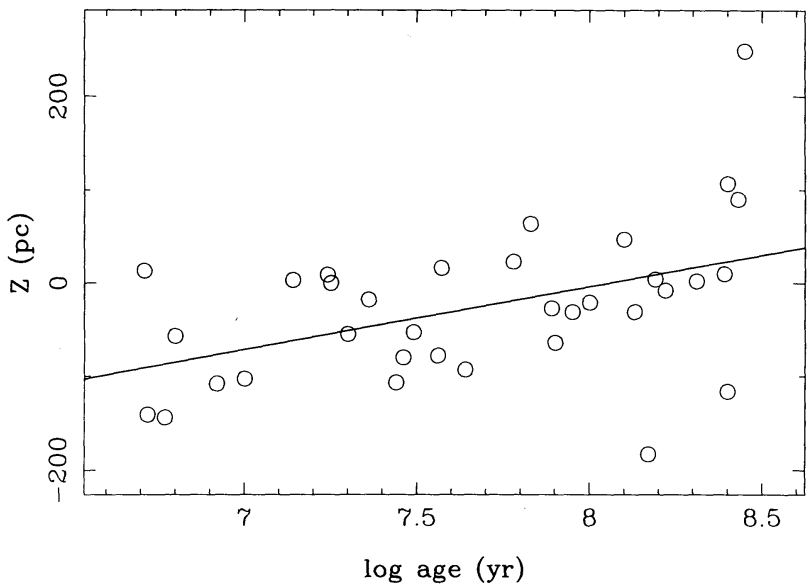

FIG. 1. $-Z$-log age diagram for 34 clusters younger than log age $=8.5$ located inside the spatial box associated with the Big Dent (see text). Linear fit to the data is also shown.

the least-squares regression line has a correlation coefficient $(r$ Pearson) of 0.46 . The present values imply that the variables are correlated at a confidence level greater than $99 \%$ (i.e., the probability of getting a correlation coefficient greater than or equal to 0.46 from a random sample of 34 objects is less than $1 \%$ ). Other correlation coefficients (for different assumptions on the probability density functions of the parent distribution) have been also computed and are listed, together with their confidence levels, in Table 1. All of these correlation coefficients are significant up to the $98 \%$ confidence level.

The standard deviation of the residuals yields a value of $\sigma=71 \mathrm{pc}$, but, as is clear from Figure 1, the dispersion seems to increase with age, and clusters older than log age $=8.1$ show the largest dispersion. This behavior could be understood as a result of kinematical dispersion in the disk, which tends to displace clusters from the original formation places in a random way. The trends in Figure 1 seem to indicate that $\sim 10^{8}$ yr can be considered as a good limiting time for the operation of diffusion processes in the solar neighborhood (i.e., younger groups do not have time to dilute their structure by kinematical diffusion).

Figure 2 is the same as Figure 1 but without objects older than log age $=8.1$. This younger subsample is mainly concentrated around $l \approx 240^{\circ}$, which corresponds to the Galactic longitude of the very center of the star-gas supercomplex III detected and labeled by Efremov \& Sitnik (1988). Figure 3 shows a histogram of clusters versus Galactic longitude which, in turn, indicates that clusters associated with the Big Dent are better defined by the Galactic longitude range $215^{\circ} \leq l \leq 260^{\circ}$. Restricting the sample to these longitudes, the resulting sub-

TABLE 1

Z-log Age Correlation for Clusters LOCATED IN THE BIG DENT

\begin{tabular}{ccc}
\hline \multicolumn{1}{c}{ Correlation Coefficient } & Value & Confidence Level \\
\hline$t$-Student $\ldots \ldots \ldots \ldots \ldots \ldots \ldots \ldots \ldots$ & 2.38 & 0.98 \\
$F \ldots \ldots \ldots \ldots \ldots \ldots \ldots \ldots \ldots \ldots \ldots$ & 22129 & 1.00 \\
$D$-Kolmogorov-Smirnov $\ldots \ldots \ldots$ & 0.70 & 1.00 \\
$r$-Pearson $\ldots \ldots \ldots \ldots \ldots \ldots \ldots \ldots \ldots$ & 0.46 & 0.99 \\
$R$-Spearman $\ldots \ldots \ldots \ldots \ldots \ldots \ldots \ldots$ & 0.40 & 0.98 \\
$\tau$-Kendall $\ldots \ldots \ldots \ldots \ldots \ldots \ldots \ldots$ & 0.32 & 0.99 \\
\hline
\end{tabular}

NoTE-Clusters younger than log age $(\mathrm{yr})=8.5 ; N=34$ objects. 


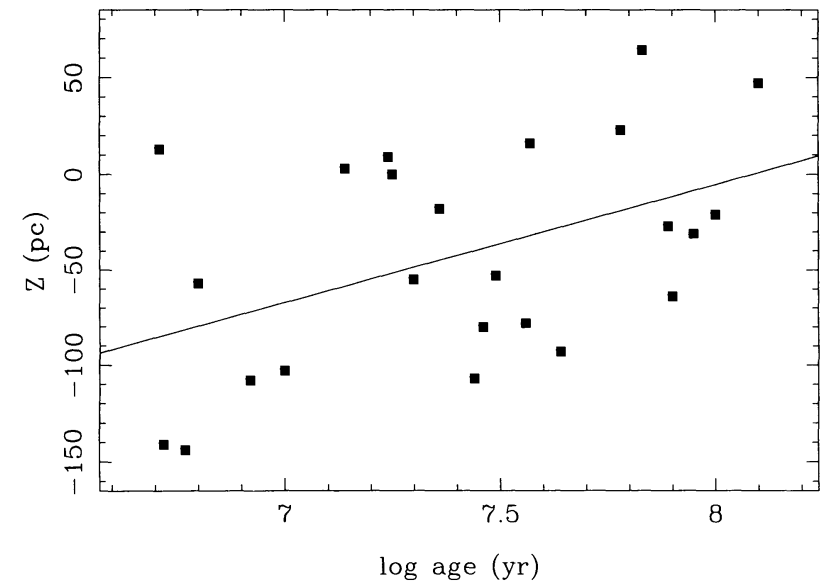

FIG. 2. $-Z$-log age diagram for 24 clusters younger than log age $=8.1$ located inside the spatial box associated with Big Dent (see text). Linear fit to the data is also shown.

sample contains 20 objects, and the $Z$-log age correlation is displayed in Figure 4. The correlation coefficient ( $r$-Pearson) between both variables now increases to 0.6 , at a significance level larger than $99 \%$. Other estimated coefficients assuming normal distributions for both variables ( $t$-Student, $F, D-$ Kolmogorov-Smirnov) and independent of the probability density function underlying the data $(r$-Pearson, $R$-Spearman, $\tau$-Kendall) are given in Table 2.

As a check for systematic errors, the behavior of $b$ (Galactic latitude) with age was also tested. The result is displayed in Figure 5, where a strong correlation between both variables also appears. The resulting correlation coefficients are given in Table 3, together with their confidence levels. The very existence of a correlation between $b$ versus log age for the clusters in this Galactic region seems to discard the possibility that the relationship has been originated by systematic errors in the distance estimates. Thus, one can conclude that open clusters associated with the star-gas supercomplex III show an age stratification in the $Z$-coordinate.

\subsection{Cepheid Stars}

Cepheids are primary calibrators for the extragalactic distance scale because they are intrinsically bright, easy to detect,

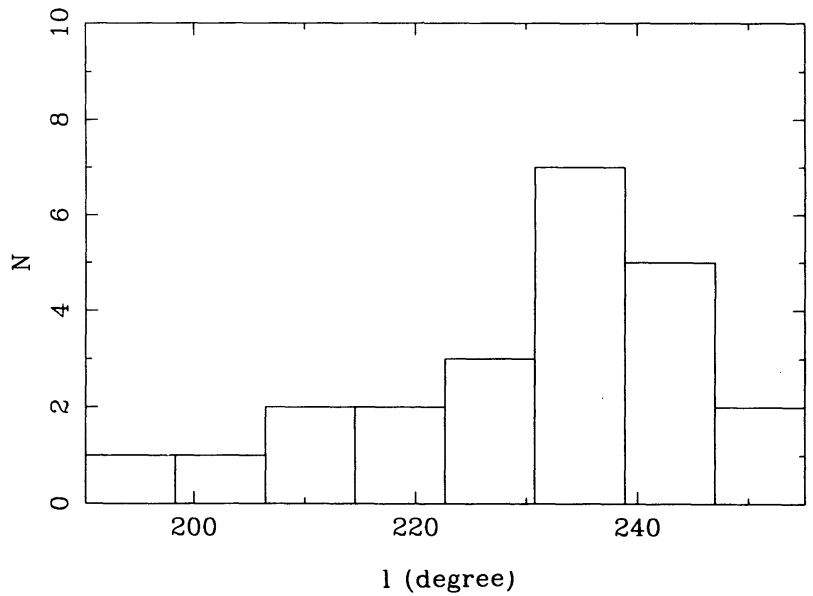

FIG. 3.-Histogram in $l$ (Galactic longitude) for the clusters located inside the spatial box associated to the Big Dent.

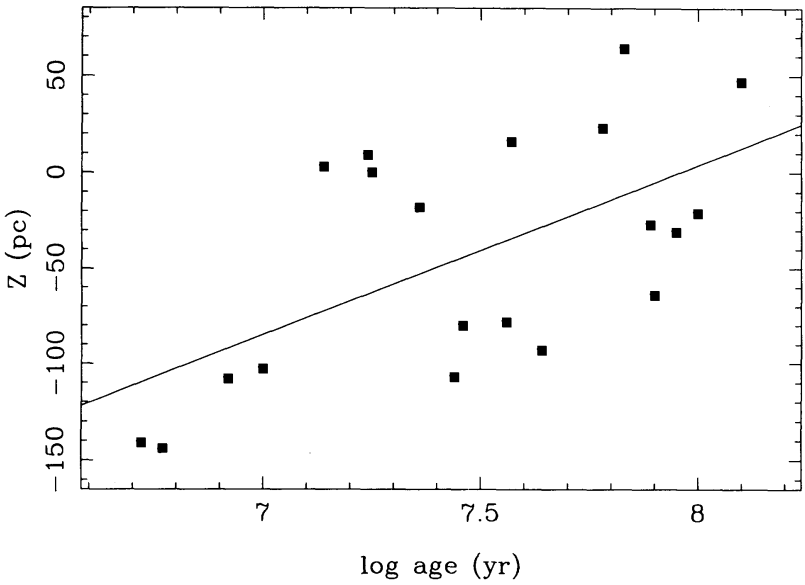

Fig. 4. $-Z$-log age diagram for 20 clusters younger than log age $=8.1$ and Galactic longitudes in the range $260^{\circ} \leq l \leq 215^{\circ}$ located in the Big Dent (see text). The linear fit to the data is also shown.

and obey a well-established period-luminosity relation. Stellar physics also provides a good theoretical relationship between pulsational periods and ages (Kippenhan \& Smith 1969), and this relationship has been already calibrated empirically (Efremov 1978b). Thus, one can get reliable distances and ages from complilations of pulsation periods and broadband photometry for these objects.

Given that Cepheids are also good probes for the location of supercomplexes (Efremov 1993), we have examined their population in the third Galactic quadrant to analyze their vertical distribution. Distances have been estimated using the periodluminosity calibration given in the catalogs, whereas the ages were computed from the empirical calibration given by Efremov (1978b). We have selected those stars located in the range of Galactic longitudes between $l=215^{\circ}$ and $l=260^{\circ}$ and contained within the same $X-Y$ box used in the open cluster analysis. Fourteen and 12 stars were selected in this way from the B87 and CC87 catalogs, respectively. These stars have ages between 3 and $8 \times 10^{7} \mathrm{yr}$, a very narrow interval, and they can be used to verify only the mean location of open clusters within this range of ages. The normal points defined as the medians of the log age and $Z$ variables for both Cepheid samples are displayed in Figure 6 (open circles) together with the open cluster data. This shows that the Cepheid data support the existence of the age gradient along the $Z$-axis.

Finally, following the referee's suggestion, we now address the question of whether a peculiar distribution of absorbing clouds along the line of sight could be causing the observed correlation.

TABLE 2

Z-log Age Correlation for Clusters LOCATED IN THE BIG DENT

\begin{tabular}{|c|c|c|}
\hline Correlation Coefficient & Value & Confidence Leve \\
\hline$t$-Student . & 3.64 & 1.00 \\
\hline 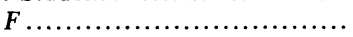 & 21799 & 1.00 \\
\hline D-Kolmogorov-Smirnov...... & 0.75 & 1.00 \\
\hline$r$-Pearson $\ldots \ldots \ldots \ldots \ldots \ldots \ldots$ & 0.60 & 0.99 \\
\hline$R$-Spearman $\ldots \ldots \ldots \ldots \ldots \ldots$ & 0.52 & 0.98 \\
\hline$\tau$-Kendall $\ldots \ldots \ldots \ldots \ldots \ldots \ldots$ & 0.40 & 0.99 \\
\hline
\end{tabular}

NoTE.-Clusters younger than $\log$ age $(y r)=8.1$ in the Galactic longitude range $215^{\circ} \leq l \leq 260^{\circ} ; N=20$ objects. 


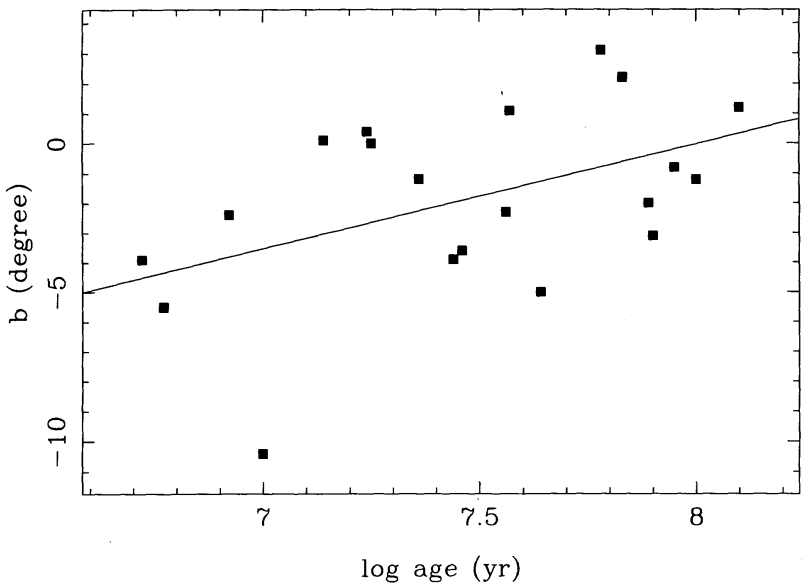

Fig. 5.- $b-\log$ age diagram for the same cluster sample as Fig. 4

\subsection{Extinction}

Given that young objects are usually associated with large amounts of extinction, absorbing clouds could, in principle, be responsible for spurious correlations between ages and locations. To check this possible problem, Figure 7 displays the cluster color excess values (from LMG94) versus log age, $Z, b$, and distances from the Sun. Except for the well-known correlation between color excess and distance, these plots show no apparent correlation between $E(B-V)$ and any other variable. Most clusters in our sample have color excesses below 0.3 , and only four objects reach $E(B-V)$ values of about 0.5 . If we assume that the location of those clusters showing the largest color excess indicates the position of dark absorbing clouds, we can conclude that only two dust layers could be present in this Galactic region. One should be located at about $1 \mathrm{kpc}$ from the Sun, and the other should be some $2 \mathrm{kpc}$ away. Their angular sizes and reddening effects, however, should be rather small. Given the large extent of the Big Dent, they are unable to affect the observed gradient heavily.

In addition, interstellar extinction models (Neckel \& Klare 1980; Arenou, Grenón, \& Gómez 1992; Vardanyan, Ambaryan, \& Pogosyan 1993) display the lowest absorption values in the directions of the third Galactic quadrant. In particular, the Puppis region $\left(l=245^{\circ}\right)$ has remarkable low interstellar absorption values, making this area very suitable for optical studies of the outer Galaxy (Orsatti 1992). We have computed the $E(B-V)$ values for different Galactic latitudes and distances in this quadrant using the code developed by Arenou et al. (1992), and these estimates do not show any appreciable variation of the extinction with $b$ at any given distance.

TABLE 3

$b$ - $\log$ Age Correlation for Clusters LOCATED IN THE BIG DENT

\begin{tabular}{crc}
\hline \hline Correlation Coefficient & Value & Confidence Level \\
\hline$t$-Student $\ldots \ldots \ldots \ldots \ldots \ldots \ldots \ldots$ & 14.26 & 1.00 \\
$F \ldots \ldots \ldots \ldots \ldots \ldots \ldots \ldots \ldots$ & 54.79 & 1.00 \\
$D-$ Kolmogorov-Smirnov $\ldots \ldots$. & 1.00 & 1.00 \\
$r$-Pearson $\ldots \ldots \ldots \ldots \ldots \ldots \ldots \ldots$ & 0.47 & 0.96 \\
$R$-Spearman $\ldots \ldots \ldots \ldots \ldots \ldots \ldots$ & 0.43 & 0.94 \\
$\tau$-Kendall $\ldots \ldots \ldots \ldots \ldots \ldots \ldots \ldots$ & 0.30 & 0.93 \\
\hline
\end{tabular}

NoTE.-Clusters younger than log age $(\mathrm{yr})=8.1$ in the Galactic longitude range $215^{\circ} \leq l \leq 260^{\circ} ; N=20$ objects.

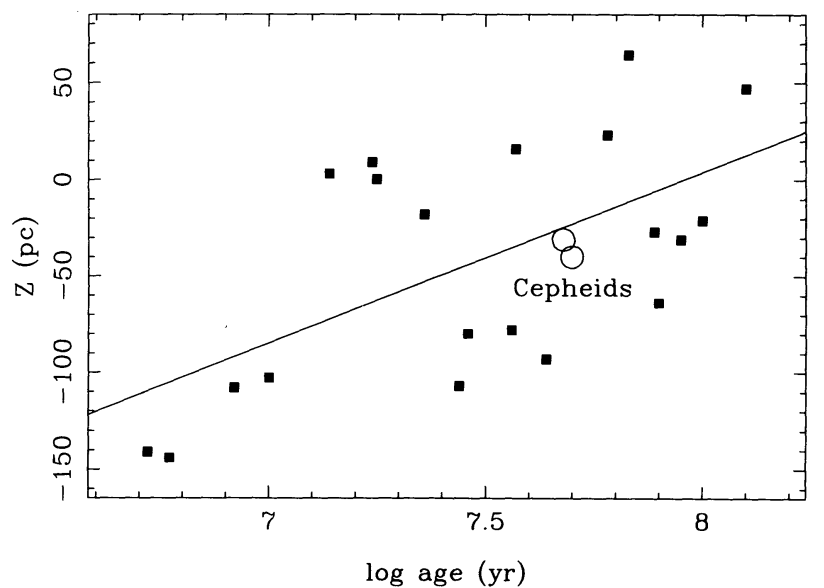

FIG. 6.-Z-log age diagram for the same cluster sample as Fig. 4 showing the median of $Z$ and log age for the Cepheid stars (B87 and CC87 catalogs; open circles) associated with the Big Dent.

Finally, the distribution of molecular clouds in the direction of the Big Dent follows the same trends indicated by the young clusters and does not show any additional peculiar features. Sodroski (1991) studied the physical properties of giant molecular cloud complexes (GMCs) in the outer Galaxy and derived distances for 35 of these objects. Figures 5 and 6 in that paper display the distribution of cloud complexes on the Galactic plane and a plot of $Z$ versus $l$, respectively. There are several complexes, numbered $51-60$ in his catalog, located in the direction of the Big Dent. Except for the complex labeled 54 , which has an estimated distance above $3 \mathrm{kpc}$, the spatial distribution of these clouds is similar to the one displayed by the open cluster sample. So, it seems that these molecular cloud complexes are also associated with supercomplex III, and, more important, their vertical distribution follows the one displayed by the younger clusters in our sample (i.e., the distribution of molecular clouds in the Big Dent simply follows the large-scale pattern displayed by the stellar population).

In summary, the observed age stratification along the $Z$-axis is not a result of a peculiar interstellar extinction pattern. Instead, it can be considered as a well-established observational fact and provides an important record of the way the stars were formed in this Galactic region.

\section{A MODEL FOR THE AGE GRADIENT}

\subsection{HVC Collisions: The Formation of Star-forming Clouds}

The collisions between HVC and the gaseous disk, which are a rich potential source of energy and momentum for the interstellar gas, can produce strong perturbations and large interstellar structures with peculiar displacements in the $Z$-axis (Tenorio-Tagle 1980; Tenorio-Tagle et al. 1986, 1987; Franco et al. 1988; Comerón \& Torra 1992; Lepine \& Duvert 1994). The shock produced by an HVC-galaxy collision, which is highly radiative, accelerates the interstellar gas in a thin shocked layer until the HVC has been completely shocked. Afterward, depending on its internal pressure and self-gravity, the layer can reexpand and merge with the ambient medium or can be held together by self-gravitational forces. Given the wide range of HVC sizes and velocities (see Kamphuis 1993), the resulting structures can range from simple velocity perturbations of a localized interstellar region to apparent "holes" in the gaseous disk, with large molecular clouds displaced from 

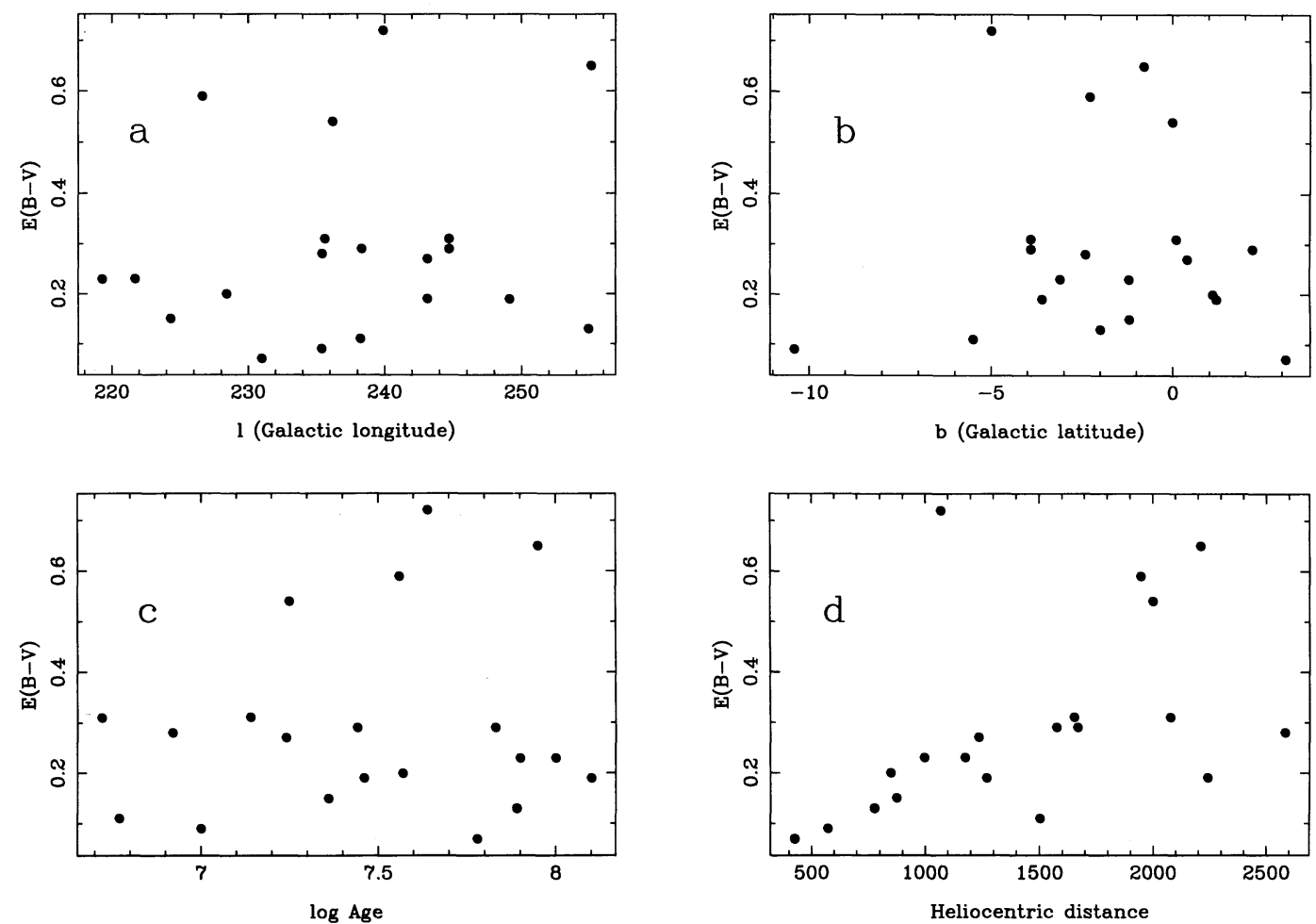

Fig. 7.-Extinction pattern in the third Galactic quadrant. The data have been taken from the LMG94 open cluster catalog. $(a) E(B-V)$ vs. $l ;(b) E(B-V)$ vs. $b$; (c) $E(B-V)$ vs. $\log$ age; $(d) E(B-V)$ vs. distance.

the midplane. Thus, the existence of star-forming regions located at large $Z$ distances from the midplane cloud be logically explained by this mechanism.

The creation of molecular clouds in the solar neighborhood requires swept-up column densities in excess of $\sim 5 \times 10^{20}$ $\mathrm{cm}^{-2}$, which is the minimum value for either high opacity or self-gravity at the solar circle (Franco \& Cox 1986). Numerical models in two dimensions for the solar neighborhood (Franco et al. 1988) indicate that such column densities can be gathered by colliding $\mathrm{HVC}$ which inject a momentum per unit area above

$$
N_{c} V_{c}=3 \mu^{-1} a_{s} \int_{0}^{\infty} \rho(Z) d Z \simeq 1.5 \times 10^{22} \mathrm{~cm}^{-2} \mathrm{~km} \mathrm{~s}^{-1}
$$

where $N_{c}$ is the HVC column density, $V_{c}$ is its velocity, $\mu$ is the mass per gas particle, $a_{s}$ is the average sound speed in the interstellar medium, and $\rho(Z)$ is the disk density distribution in the $Z$-direction. The average sound speed is assumed equal to the cloud-cloud velocity dispersion among the "normal" population of diffuse $\mathrm{H}$ I clouds, $a_{s} \sim 8 \mathrm{~km} \mathrm{~s}^{-1}$ (e.g., Kulkarni $\&$ Fich 1985), and the main gaseous disk is represented by a single Gaussian distribution

$$
\rho(z)=\rho(0) e^{-Z^{2} / H^{2}},
$$

with a midplane gas density $\rho(0)=5 \times 10^{-24} \mathrm{~g} \mathrm{~cm}^{-3}$ and an effective height $h=\pi^{1 / 2} H / 2=10^{2}$ pc.

For these types of collisions, the shocked layer becomes a self-gravitating structure when it reaches a column density

$$
N_{\mathrm{sg}} \simeq 1.2 \times 10^{21} \mathrm{~T}_{3} r_{2}^{-1} \mathrm{~cm}^{-2}
$$

where $T_{3}$ is the shocked layer temperature in units of $10^{3} \mathrm{~K}$, and $r_{2}$ is its radius in units of $10^{2} \mathrm{pc}$. Given the typical param- eters involved (i.e., the column density of the half-disk and the properties of the colliding $\mathrm{HVC}$ ), the transformation of the shocked layer into a star-forming cloud is better achieved for large impinging clouds, with $r_{2} \geq 1$. Once the shocked layer becomes a self-gravitating cloud, its dynamical evolution can be approximated by the motion of its center of mass (large interstellar structures could be disrupted into several pieces by tidal forces, but each one of these pieces follows the same evolutionary path; see Franco et al. 1988).

\subsection{Motion of the Star-forming Cloud}

The resulting cloud, which eventually becomes a starforming cloud, maintains its velocity in the $Z$-direction, and its further evolution is controlled by the local gravitational field and its interaction with the ambient medium. Given that the cloud is self-gravitating, here we assume that its motion can be described by the evolution of its center of mass. Considering only the motion along the $Z$-axis, the forces acting on the cloud are the disk gravity, the drag from the ambient medium, radiation pressure from the ambient stellar disk, and the pressure (radiative and mechanical) generated by the new stars formed within the cloud. The mechanical energy injected via stellar winds and supernova explosions in a newly formed cluster is similar to its total radiative energy (e.g., Abbot 1982; Leitherer, Robert, \& Drissen 1992). Thus, for simplicity, we include the effects of both types of energy in a single pressure term.

Radiation pressure on dust grains is very efficient in driving, via gas-grain coupling, a variety of interstellar flows, and starlight can "photolevitate" small interstellar clouds to high Galactic latitudes (see Franco \& Ferrara 1992; Dwek \& Arendt 1992). Here we solve the equation of motion with a 
radiation pressure term and assume that the effects of the mechanical term can be included by simply increasing the average cluster luminosity by a factor of 3 . The overall effect of the ambient radiation field is small for cloud column densities above $\sim 5 \times 10^{20} \mathrm{~cm}^{-2}$, but the pressure due to a luminous newly born stellar cluster can certainly modify the evolution (see Franco et al. 1991). The direction of the radiative force depends on the relative location of the new cluster. Given that the stars are formed downstream, in the regions compressed at early times, a fraction of them can detach from the cloud and fall down toward the plane. In addition, star formation erodes the cloud on one side and sends waves upstream that can trigger further star formation (i.e., Elmegreen \& Lada 1977). Once the parental cloud cores are dispersed by expanding $\mathrm{H}$ II regions (see Franco, Shore, \& Tenorio-Tagle 1994), the rest of the stellar cluster also falls down toward the plane. For simplicity, then, we assume that the recently formed star clusters occupy different $Z$-layers, each one with its own cluster surface density $\sigma_{i}$. Also, given the large size of the Big Dent, the separation of the cloud from each layer is small compared with the layer extension, and one can approximate the upward radiation pressure as

$$
P_{\mathrm{rad}}=\frac{\left(1-e^{-\tau}\right)}{2 c} \int Q_{p}(v) L(v) d v \sum_{i} \sigma_{i},
$$

where $\tau$ is the cloud optical depth, $c$ is the speed of light, $L(v)$ is the luminosity of a cluster at frequency $v$, and $Q_{p}(v)$ is the efficiency factor for radiation pressure.

The resulting equation of motion can be written in a simple manner (Franco et al. 1991):

$$
\dot{v}_{z}=g_{z}-\frac{\rho(0) e^{-z^{2} / H^{2}} U_{z}}{N \mu}+\frac{P_{\mathrm{rad}}}{N \mu},
$$

where $g_{z}$ is the gravitational acceleration along the $Z$-axis, $N$ is the cloud column density along the $Z$-axis, $\mu$ is the mass per gas particle, and $U_{z}$ is a function depending on the cloud shape and velocity. For subsonic motions with laminar streamlines flowing around the cloud, $U_{z}$ can be approximated by $a_{s} v_{z}$, where $a_{s}$ is the sound speed. Otherwise, for supersonic motions or turbulent flows, it may be better approximated by $v_{z}^{2}$. In addition, for the supersonic case, the cloud column density increases as the shock front collects the interstellar gas, and we follow the evolution of $N$ and solve equation (5) with $N$ variable. This equation of motion neglects the compression generated by the radiative and drag forces and assumes that the cloud as a whole receives the effects of $P_{\text {rad }}$ and the drag. In reality, $P_{\text {rad }}$ operates up to column densities with $\tau \sim 1$, and the drag compresses only the outer layers (driving sonic waves inward which eventually reduce $v_{z}$ ).

\subsection{A Model for the Big Dent}

The equation of motion (5) can be easily solved by numerical integration. The gravitational force in the $Z$ direction is computed with a simple fit to Oort's (1960) curve. The function $U_{z}$, as discussed in the previous section, is taken as $a_{s} v_{z}$ when the motion is subsonic and as $v_{z}^{2}$ for the supersonic case. The radiation pressure term requires knowledge of the cluster surface densities as a function of time, $\sigma_{i}(t)$, and a few additional assumptions have been made. Given that new stars are continuously formed in the cloud, the term $\int Q_{p}(v) L(v) d v \sum_{i} \sigma_{i}=$ $L_{c} \bar{Q}_{p} \sum \sigma_{i}$ in equation (4) is expected to be an increasing function of time. For simplicity, here we assume a linear time dependence

$$
L_{c} \bar{Q}_{p} \sum_{i} \sigma_{i}=\beta t
$$

where $t$ is the time and $\beta$ is a constant. The value of this constant is obtained by defining an age to the Big Dent and using some of its properties.

The $Z$-age relationship indicates that the Big Dent was probably already forming stars some $3-4 \times 10^{7} \mathrm{yr}$ ago, and, for simplicity, we assume that the star-forming cloud passed through the plane some $4 \times 10^{7}$ yr ago (i.e., half of the period of oscillation about midplane). The total mass of the cloud is about $10^{7} M_{\odot}$ (see below), and assuming a $10 \%$ star-forming efficiency (which is slightly above the average value for the Galaxy: see Franco et al. 1994), the total mass in newly formed star clusters becomes about $10^{6} M_{\odot}$. The total number of clusters formed in the Big Dent is difficult to derive directly from the catalogs (the number of clusters in the Loktin \& Matkin and LMG94 compilations is 34 , but there are also many additional $O B$ stars and $H$ II regions within in the structure), and we simply estimate the energy output from the total stellar mass. Assuming a Salpeter initial mass function with an upper stellar mass limit of $120 \mathrm{M}_{\odot}$ (see Leitherer et al. 1992), the luminosity of $10^{6} M_{\odot}$ in stars during the mainsequence phase is about $8 \times 10^{8} L_{\odot}$. As stated before, to include the effects of the mechanical energy, we increase the "effective" luminosity to $\sim 2.4 \times 10^{9} L_{\odot}$. At the present age of the structure, the total surface area is about $4 \mathrm{kpc}^{2}$, and this gives $L_{c} \bar{Q}_{p} \sum_{i} \sigma_{i} \sim 6 \times 10^{8} L_{\odot} \mathrm{kpc}^{2}$ at $t=4 \times 10^{7} \mathrm{yr}$. Given that differential rotation is continuously increasing the area of the structure, the cluster density was obviously larger in the past. Here we assume a HVC with an initial radius of $400 \mathrm{pc}$ (see below); thus the initial area of the structure is $0.5 \mathrm{kpc}^{2}$, and the maximum cluster density value becomes some 8 times larger than the present value. With these values, and writing $\sigma_{i}$ in units of $\mathrm{kpc}^{-2}$, the constant becomes

$$
\beta=\left(\frac{L_{c} \bar{Q}_{p}}{10^{6} L_{\odot}}\right)\left(\frac{t}{10^{7} \mathrm{yr}}\right)^{-1} \sum_{i} \sigma_{i} \sim 1200 \mathrm{kpc}^{-2} .
$$

We begin the numerical integration at $t=0$, when the shocked cloud is passing through midplane, and take its initial column density to be $N_{0}=9 \times 10^{20} \mathrm{~cm}^{-2}$ (corresponding to a HVC column density of $3 \times 10^{20} \mathrm{~cm}^{-2}$ ) with an initial velocity of $v_{z}(0)=25 \mathrm{~km} \mathrm{~s}^{-1}$. These initial values are above the critical values defined in equation (1) and give good results, as can be seen in Figure $8 a$. The mass of the original HVC depends on its initial column density and assumed cloud geometry. For a cylindrical cloud with radius $\sim 400 \mathrm{pc}$ and a column density of $3 \times 10^{20} \mathrm{~cm}^{-2}$, the resulting mass is about $10^{6} M_{\odot}$. This value is certainly modest compared with the $\mathrm{H}$ I gas masses derived by Kamphuis (1993) for the largest HVC detected in NGC 6946 (several times $10^{7} M_{\odot}$ ) but cannot be compared with the masses of the population of Galactic HVCs (because the distances are unknown). The resulting mass of the Big Dent parental cloud is about $10^{7} M_{\odot}$.

The motion of the cloud (more properly, a typical section of it) is given by the thick line; other lines give the motion of clusters born from the cloud at different times (with a numbered cross on the thick line). The present time corresponds to $\sim 3.9 \times 10^{7} \mathrm{yr}$. This solution indicates that star clusters younger than $10^{7}$ yr should be located at distances from the midplane greater than $\sim 120 \mathrm{pc}$, whereas clusters formed some $1.5 \times 10^{7} \mathrm{yr}$ ago should have $Z$-locations of about $80 \mathrm{pc}$. The 


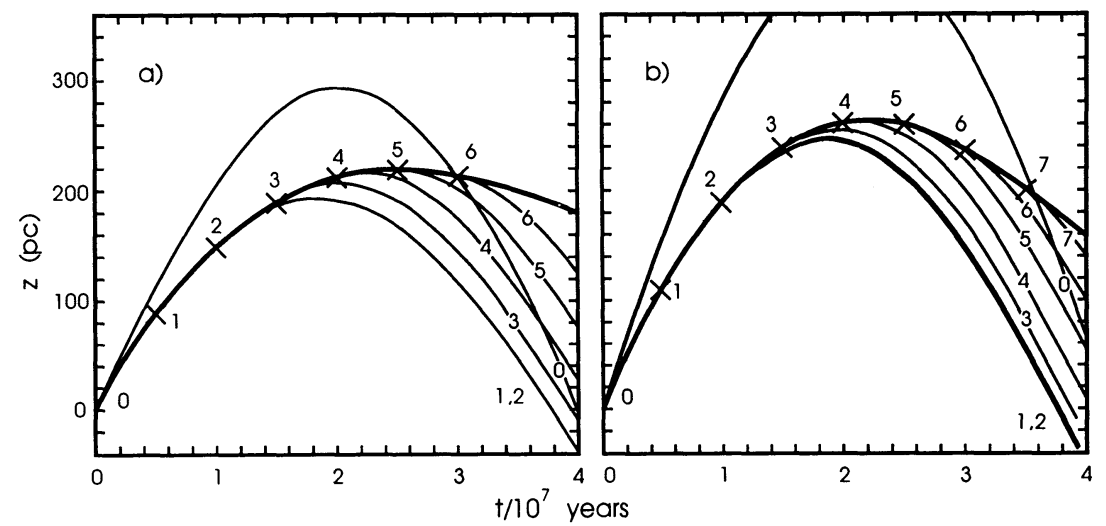

Fig. 8.-Evolution of the star-forming cloud and stellar groups generated at different epochs. The thick line represents the evolution of the cloud. The numbered thin lines display the evolution of stellar groups generated every $5 \times 10^{6} \mathrm{yr}$ (the times of birth are represented by the crosses). (a) Continuous star formation with $v_{z}(0)=25 \mathrm{~km} \mathrm{~s}^{-1}$. (b) Pulsed star formation with episodes every $5 \times 10^{6} \mathrm{yr}$ and $v_{z}(0)=30 \mathrm{~km} \mathrm{~s}^{-1}$.

population older than $2-3 \times 10^{7} \mathrm{yr}$, on the other hand, has already merged with the rest of the stellar disk, and it is within a thickness of 20-40 pc (note that except for clusters born around $t=0$, all clusters exert an upward radiation force on the cloud). Equation (6) assumes a continuous formation of clusters. We can consider a discontinuous case in which star clusters are formed every $5 \times 10^{6} \mathrm{yr}$ but still at the rate given by equation (6) (at intermediate times the radiative flux is constant). Figure $8 b$ shows the results for this model with episodic star formation. Because of the smaller efficiency of the radiation thrust in this case, a higher $v_{z}(0)$ is now needed to reach $Z$-distances similar to the ones displayed in Figure $8 a$. The results shown in Figure $8 b$, however, are basically the same as those in Figure $8 a$. Figure 9 represents a reasonable fit of the model (Fig. 8a) to the age gradient of the Big Dent, the main role being played by the radiation force, retarding the fall of the cloud.

\section{DISCUSSION}

One of the fundamental aims in studying the properties of large-scale star formation is to understand the link between the star-forming processes and the structural properties of the host galaxy. Star formation in spiral galaxies often appears to occur in large gas complexes, with characteristic sizes of the order of

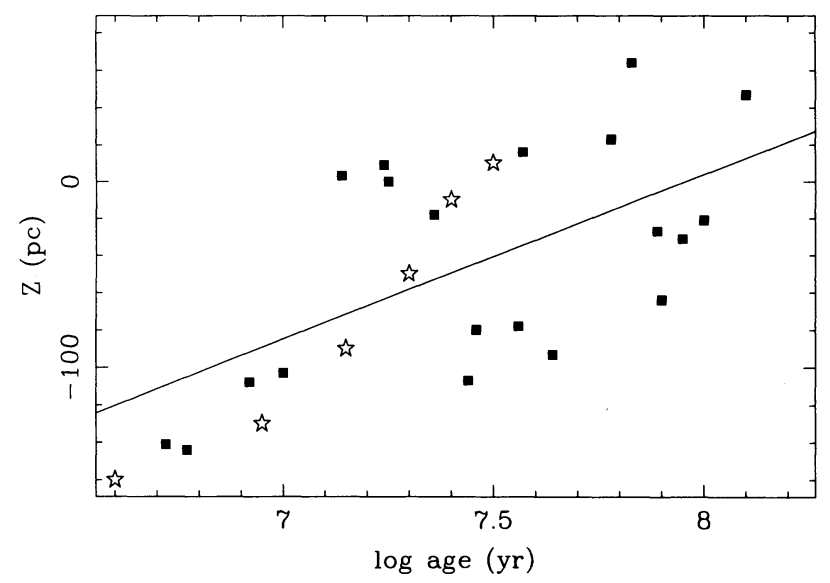

FIG. 9.-Z-log age diagram for the same cluster sample as Fig. 4, including the values estimated from model (stars) assuming a continuous formation of clusters.
$1 \mathrm{kpc}$ and masses about $10^{7} M_{\odot}$, aligned along, or in segments of, spiral arms (e.g., Efremov 1978a; Elmegreen \& Elmegreen 1983; Larson 1988). Thus, these large star-forming complexes represent the largest coherent scale of star formation in spiral and irregular galaxies, becoming a natural target for the study of triggered and propagated star formation (e.g., Palouš, Tenorio-Tagle, \& Franco 1994). The relative location between the loci of star formation and the spiral pattern has suggested that spiral density waves drive or trigger (to some degree) the birth of new stars in the Galactic disk. However, some of the physical properties observed in intermediate- and large-size star-forming complexes depart from those expected for a star formation history (mainly) based on density wave theory. The kinematics of some star-forming complexes in the vicinity of the Sun (some 3-4 kpc) shows a velocity field incompatible either with a circular model or with that originated by a density linear model (Avedisova \& Palouš 1989; Phelps 1992). In addition, the current accuracy in determining the star formation efficiency does not enable us to establish a clear link between spiral arm and star formation triggering (M51 is a typical example of this controversy; Vogel, Kulkarni, \& Scoville 1988; Rand \& Kulkarni 1992; Knappen et al. 1992; Elmegreen 1993).

The relative location of gas and young stellar objects, with respect to the midplane, could also be an important clue to understanding the properties of large-scale star formation in our Galaxy. Several examples of these large $Z$-departures (some of them are associated with star-forming regions) can be found in the literature: The Orion and Monoceros molecular clouds (Franco et al. 1988), the Cepheus bubble (Kun, Balazs, \& Tóth 1987), H I chimney-type structures (Heiles 1984), and “worms" (Heiles 1989; Li \& Ikeuchi 1989, 1990). A detailed analysis of the three-dimensional spatial distribution of young open clusters has shown that the four nearest supercomplexes are located below the Galactic plane (Alfaro et al. 1991, 1992). This surprising result indicates that aside from the action of spiral waves, there are some $Z$-displacements, or oscillations, naturally connected to the processes of star formation. Our Galaxy is not a closed system, and the halo-disk interaction is continuously modifying the physical properties of the Galactic disk (e.g., Mirabel 1991; Larson 1993 and references therein). The exchange of mass and energy between the halo and the disk (in both directions) should largely affect, among other things, the chemical evolution, the rate of star formation, and 
the dynamical and spatial structure of the disk. In this way, HVCs can be considered as the transmission vectors for this exchange and led Alfaro et al. (1991) to propose that the largest depression detected in the solar neighborhood (the Big Dent) was, probably, originated by a HVC-disk collision. Given the mass gathered in such a collision, this event could have also triggered a series of episodes of star formation.

The results presented in $\S 2$ show the existence of an age gradient in the Big Dent and indicate the existence of a complicated internal structure in supercomplexes. This age stratification in the $Z$ coordinate was also corroborated with the available Cepheid data and seems to be free of extinction effects. Obviously, these results must be considered as a first approach to a detailed study of the internal structure in supercomplexes, and further detailed studies should shed more light on their star formation history.

We have also modeled the kinematics of a cloud generated by a HVC-disk collision including both the interstellar drag and radiative pressure. The effective radiative force on the cloud is produced by newly formed star clusters. These clusters may be assumed to form either continuously or at a series of different time intervals. The selection of the (small) time interval within successive bursts of star formation is not strongly relevant for the model. The model parameters (i.e., the initial cloud column density, initial cloud velocity, initial cluster luminosity, etc.) are selected to fall within a "reasonable" range of values (e.g., Franco et al. 1988; Franco et al. 1991). The solu- tion shown in Figure 9 provides an age gradient which is in reasonable agreement with the observed values and indicates that a HVC-disk collision is certainly able to explain the morphology and structure of the Big Dent. A key element in the model is the "levitation" generated by radiative pressure which is responsible for the gradient. The radiation force is directed away from the Galactic plane and is mainly produced by those clusters located near the cloud. In computing the motion of a vertical section of the cloud, we have represented this force as produced by the different layers of clusters born from the cloud. Thus, each generation of star clusters in the Big Dent is assumed to emerge from the shocked cloud and evolves in the gravitational field of the disk, generating the observed gradient. The model predicts that the high-latitude clusters should move toward the plane, and further studies of the cluster space velocities will help to clarify this issue.

We thank F. Arenou for kindly providing us with his code to compute the extinction in the Galactic disk. We also thank an anonymous referee for a series of very challenging and certainly useful suggestions. E. J. A. and J. C. C. acknowledge the financial support from Junta de Andalucía (PAI) and from DIGICYT (Spain) through the project PB91-0053. Part of this work was performed during the sabbatical term of E. J. A. at Boston University. J. F. and E. M. acknowledge the support given to this project by DGAPA-UNAM through the grant IN103991.
Abbot, D. C. 1982, ApJ, 263, 723

Alfaro, E. J., Cabrera-Caño, J., \& Delgado, A. J. 1991, ApJ, 378, 106 1992, ApJ, 399, 576

Arenou, F., Grenon, M., \& Gómez, A. 1992, A\&A, 258, 111

Avedisova, V. S., \& Palouš, J. 1989, Bull. Astron. Inst. Czechoslovakia, 40, 42

Berdnikov, L. N. 1987, Variable Stars, 22, 505 (B87)

Berdnikov, L. N., \& Efremov, Yu. N. 1993, Astron. Lett., 19, 389

Cadwell, J. A. R., \& Coulson, I. M. 1987, AJ, 93, 1090 (CC87)

Comerón, F., \& Torra, J. 1992, A\&A, 261, 94

Dwek, E., \& Arendt, R. G. 1992, ARA\&A, 30, 11

Efremov, Yu. N. 1978a, Soviet Astron. Lett., 4, 6 1978b, Soviet Astron., 22, 161

1993, in Star Formation, Galaxies, and The Interstellar Medium ed.

J. Franco, F. Ferrini, \& G. Tenorio-Tagle (Cambridge: Cambridge Univ. Press), 360

Efremov, Yu. N., \& Sitnik, T. G. 1988, Soviet Astron. Lett., 14, 347

Elmegreen, B. G. 1992, in Star Formation in Stellar Systems, ed. G. Tenorio-

Tagle, M. Prieto, \& F. Sanchez (Cambridge: Cambridge Univ. Press), 381

Tagle, M. Prieto, \& F. Sanchez (Cambridge: Cambridge Univ. Press), 381

J. Franco, F. Ferrini, \& G. Tenorio-Tagle (Cambridge: Cambridge Univ. Press), 108

Elmegreen, B. G. \& Elmegreen, D. M. 1983, MNRAS, 203, 31

Elmegreen, B. G., \& Lada, C. 1977, ApJ, 214, 725

Franco, J. 1992, in Star Formation in Stellar Systems, ed. G. Tenorio-Tagle,

M. Prieto, \& F. Sanchez (Cambridge: Cambridge Univ. Press), 515

Franco, J., \& Cox, D. 1986, PASP, 98, 1076

Franco, J., \& Ferrara, A. 1992, in Evolution of Interstellar Matter and

Dynamics of Galaxies, ed. J. Palous, W. Burton, \& P. O. Linblad

(Cambridge: Cambridge Univ. Press), 130

Franco, J., Ferrini, F., Ferrara, A., \& Barsella, B. 1991, ApJ, 366, 443

Franco, J., Shore, S. N., \& Tenorio-Tagle, G. 1994, ApJ, 436, 795

Franco, J., Tenorio-Tagle, G., Bodenheimer, P., Różyczka, M., \& Mirabel, I. F. 1988, ApJ, 333, 826

Heiles, C. 1984, ApJS, 55, 585

1989, in Structure and Dynamics of the Interstellar Medium, ed.

G. Tenorio-Tagle, M. Moles, \& J. Melnick (Berlin: Springer), 484

Janes, K. A., Tilley, C., \& Lyngå, G. 1988, AJ, 95, 77

Kamphuis, J. 1993, Ph.D. thesis, Groningen Univ.

Kippenhahn, R., \& Smith, L. 1969, A\&A, 1, 142

Knappen, J. H., Beckman, J. E., Cepa, J., van der Hulst, T., \& Rand, R. 1992

ApJ, 385, L37

\section{REFERENCES}

Kulkarni, S., \& Fich, M. 1985, 289, 782

Kun, M., Balazs, L. G., \& Tóth, I. 1987, A\&SS, 134, 211

Larson, R. B. 1988, in Galactic and Extragalactic Star Formation, ed. R. E Pudritz \& M. Fich (Dordrecht: Kluwer), 459 1993, in Star Formation in Stellar Systems, ed. G. Tenorio-Tagle,

M. Prieto, \& F. Sanchez (Cambridge: Cambridge Univ. Press), 127

Leitherer, C., Robert, C., \& Drissen, L. 1992, ApJ, 401, 596

Lepine, J. R. D., \& Duvert, G. 1994, A\&A, 286, 60

Li, F., \& Ikeuchi, S. 1989, 41, 221 1990, ApJS, 73, 401

Loktin, A. V., \& Matkin, N. V. 1990, Soviet Astron., 34, 571

Loktin, A. V., Matkin, N. V., \& Gerasimenko, T. P. 1994, The Catalogue of Galactic Cluster Parameters from UBV Data (Strasbourg: Observatorie de Strasbourg, Centre de Données Stellaires) (LMG94)

Lyngå, G. 1981, Catalog of Open Cluster Data (Strasbourg: Observatoire de Strasbourg, Centre de Données Stellaires) 1982, A\&A, 109, 213

1985 , in The Milky Way Galaxy, ed. H. van Woerden, R. J. Allen, \& W. B. Burton (Dordrecht: Reidel), 133

1987, Catalog of Open Cluster Data (Strasbourg: Observatoire de Strasbourg, Centre de Données Stellaires)

Mirabel, I. F. 1991, in IAU Symp. 144, The Interstellar Disk-Halo Connection in Galaxies, ed. H. Bloemen (Dordrecht: Kluwer), 89

Neckel, Th., \& Klare, G. 1980, A\&AS, 42, 251

Oort, J. 1960, Bull. Astron. Inst. Netherlands, 15, 45

Orsatti, A. 1992, AJ, 104, 590

Palouš, J., Tenorio-Tagle, G., \& Franco, J. 1994, MNRAS, 270, 75

Phelps, R. 1992, Ph.D. thesis, Boston Univ.

Rand, R., \& Kulkarni, S. R. 1992, ApJ, 349, 243

Sitnik, T. G. 1989, Soviet Astron. Lett., 15, 388

Sodroski, T. J. 1991, ApJ, 366, 95

Spicker, J, \& Feitzinger, J. V. 1986, A\&AS, 163, 43

Tenorio-Tagle, G. 1980, A\&A, 88, 61

Tenorio-Tagle, G., Bodenheimer, P., Różyczka, M., \& Franco, J. 1986, A\&A 170,107

Tenorio-Tagle, G., Franco, J., Bodenheimer, P., \& Różyczka, M. 1987, A\&A 179,219

Vardanyan, R. A., Ambaryan, V. V., \& Pogosyan, A. V. 1993, Astrophysics, 36, 122

Vogel, S. N., Kulkarni, S. R., \& Scoville, N. Z. 1988, Nature, 334, 402 